\title{
Part 3
}

\section{Space Debris}




\title{
The Space Debris Environment - Past and Present
}

\author{
W. Flury ${ }^{1}$ \\ ESA/ESOC, Robert Bosch Str. 5, 64293 Darmstadt, Germany \\ wflury@esa.int
}

\begin{abstract}
The mass and number of Earth-orbiting human-generated space debris have increased steadily since the beginning of space flight. Recent voluntary measures for debris mitigation applied by space operators have not stemmed the increase. The debris hazard for manned and unmanned missions is still low, but rising. More effective but also more costly measures, such as selective deorbiting of used stages, will be necessary to avoid a run-away situation. Internationally agreed codes for debris management and control are needed to solve this global space environment problem.
\end{abstract}

\section{Introduction}

Since the launch of Sputnik 1 in 1957 there have been more than 4000 successful rocket launches. Regular tracking of Earth-orbiting objects has detected nearly 25,000 objects. This space surveillance activity is carried out by the USA and by Russia using global networks of radars and optical sensors. There are currently 9,000 catalogued objects in orbit, with a total estimated mass of 2,000 tons in LEO. There are currently estimated to be more than 100,000 objects larger than $1 \mathrm{~cm}$ in orbit. Figure 1 shows the growth in catalogued debris from 1957 to 1999.

'Orbital debris is defined as any man-made Earth-orbiting object which is non-functional with no reasonable expectation of assuming or resuming its intended function or any other function for which it is or can be expected to be authorized, including fragments and parts thereof. Space debris comprises orbital debris and reentering debris.' IAA: International Academy of Astronautics

Sources of debris include defunct spacecraft (21\%), rocket upper stages $(18 \%)$, operational debris (12\%), fragments from breakups (43\%), exhaust products of solid fuel rockets and materials produced by ageing effects (e.g. particles such as paint flakes, or sodium and potassium droplets from the leaking RORSAT cooling system (Kessler et al. 1997)). In 1998 there were 6 breakups, generating

\footnotetext{
${ }^{1}$ This summary of Dr. Flury's presentation is based on the tranparencies he used. For further information, see the review paper by Flury (1998), the Technical Report on Space Debris (A/AC/105/720) of the Scientific and Technical Subcommittee of COPUOS (which Dr. Flury played a leading role in finalizing), and the UNISPACE III Report (A/CONF.184/6).
} 
more than 400 fragments, only 84 of which are catalogued. In 1999 there have already been 3 more breakups.

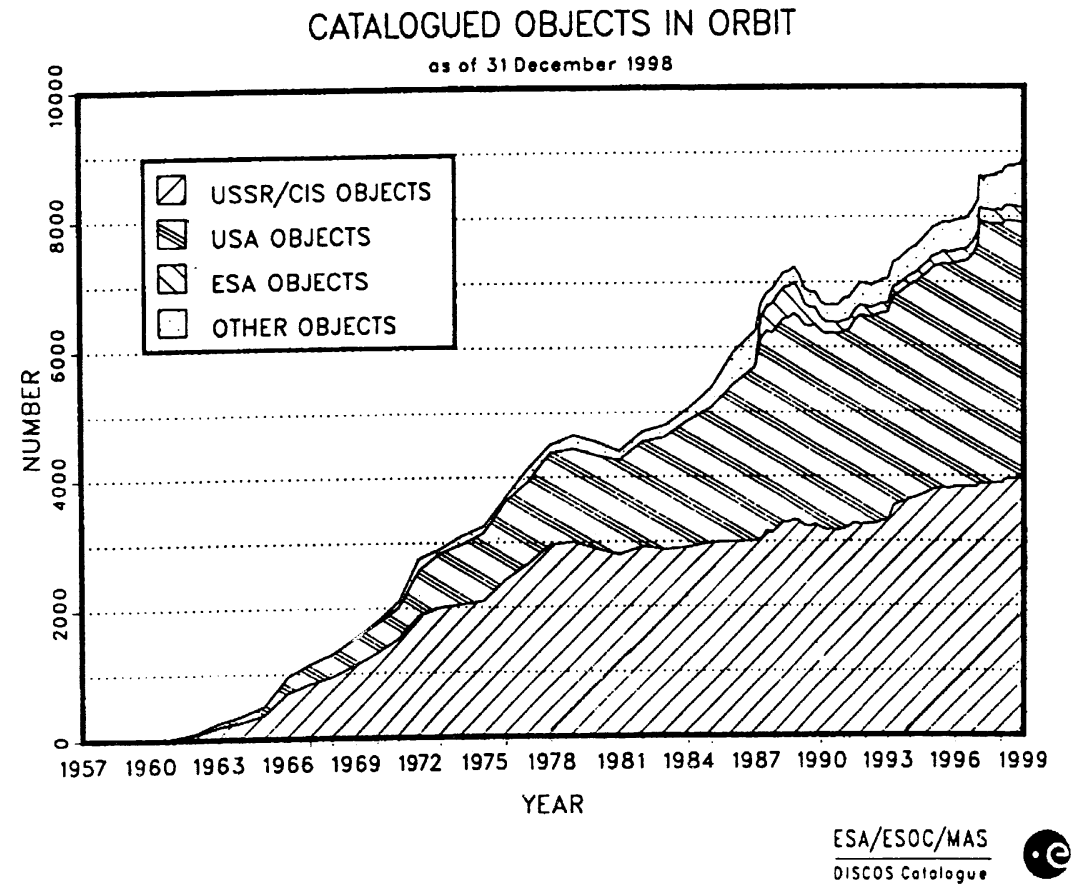

Figure 1. Catalogued objects in space since 1957. To be detected, the cbjects must be at least $\sim 10 \mathrm{~cm}$ in size in LEO and at least $\sim 1 \mathrm{~m}$ in size in GEO.

This papers summarizes the current space debris environment and outlines measures which are needed to reduce the growth of the debris population.

\section{The Situation in Low Earth Orbit (LEO) and the Geostationary Ring (GSO)}

About $85 \%$ of catalogued objects are in low Earth orbit (between $200 \mathrm{~km}$ and $2000 \mathrm{~km}$ altitude), with maxima of spatial density near $1,000 \mathrm{~km}$ and $1,500 \mathrm{~km}$ altitude. Some altitude bands may have reached critical density. More than 140 fragmentations are known to have occured in LEO. Other indicators of the degrading LEO environment include:

- Impacts on spacecraft returned from space (e.g. SolarMax, NASA's Long Duration Exposure Facility LDEF, ESA's EURECA, the solar arrays of the HST, more than 60 windows replaced on Space Shuttles, etc.)

- Evasive manoeuvres of the Space Shuttle and other spacecraft (ERS-1 and SPOT-2) 
- Collision of Cerise with fragment of H10 of Ariane V16 (July 1996)

- More than 200 shields of International Space Station (ISS)

- Trails on astronomical observations

Atmospheric drag provides a natural removal mechanism from LEO, but is effective only at the lowest altitudes.

In the geostationary ring there are about 700 catalogued objects, including about 150 upper stages of rockets. Two fragmentations are known (Titan upper stage and EKRAN spacecraft). Relative velocities are much lower than in LEO. There is no natural removal mechanism from GEO, however, so objects brought into the geostationary ring will generally remain in its vicinity indefinitely. The growth of debris in GEO is shown in Figure 2. The annual launch rate of about 25-30 spacecraft is also increasing. There is a growing trend to place several satellites in the same longitude window (colocation), due to sustained demand for orbital positions in GEO.

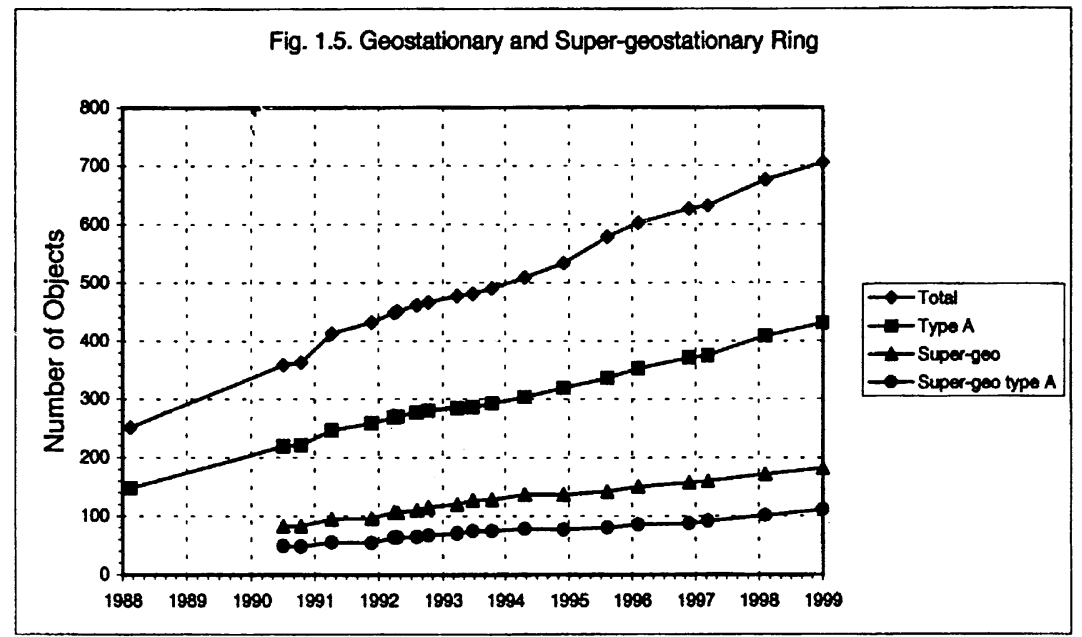

Figure 2. Catalogued objects in the geostationary ring since 1988. The objects are at least $1 \mathrm{~m}$ in size.

\section{Long Term Evolution and Debris Mitigation}

The current evolution of the debris population is determined by launch activities and breakups. If the spatial density of debris becomes large enough, population growth will be dominated by collisions and the avalanche effect will lead to uncontrolled growth. The future evolution will be strongly dependent on applied debris mitigation measures.

Current measures are directed to avoid creating new space debris by avoiding breakups, avoiding mission-related debris and use of graveyard orbits. These 
measures are in the long term not sufficient to avoid a run-away situation. Selective deorbiting of large objects (spacecraft and rocket upper stages) will become necessary. There is a high cost to deorbiting safely from high-energy orbits, without intersecting the zone of operational GSO spacecraft.

\section{Activities of International Organizations}

The Inter-Agency Space Debris Coordination Committee (IADC) was founded in 1993, with the purpose of exchanging information on space debris research, to review progress of cooperative activities, to facilitate opportunities for cooperation in debris research and to identify and evaluate mitigation options. The activities of the IADC, the IAA (International Academy of Astronautics) and other international bodies such as COSPAR have made positive contributions to space debris research and education.

Space debris was included as an agenda item for the thirty-first session of the Scientific and Technical SubCommittee of UNCOPUOS, in February 1994. A multi-year work plan was agreed at the thirty-second session. The technical report on space debris will be submitted for approval during UNISPACE-III.

\section{Conclusions}

- The space debris population is steadily growing (satellite constellations)

- Current risk levels are still low but are increasing

- Orbital regions most at risk are in LEO $(800 \mathrm{~km}-1,600 \mathrm{~km})$ and in GEO

- Most endangered regions are also the most useful regions

- Clean-up is economically not feasible: PREVENTION IS NEEDED

- Current debris reduction measures are not sufficient to avoid a run-away situation

- More efficient measures are needed, including selective deorbiting and lifetime reduction of spacecraft and rocket bodies

\section{References}

Flury, W. 1998, The Space Debris Environment of the Earth, ASP Conf. Ser. $139,49-64$

Kessler, D. J. et al. 1997, The Search for a Previously Unknown Source of Orbital Debris: the Possibility of a Coolant Leak in Radar Ocean Reconnaissance Satellites, JSC-27737, NASA JSC (Houston, USA)

Technical Report on Space Debris, Text of the Report adopted by the Scientific and Technical Subcommittee of the United Nations Committee on the Peaceful Uses of Outer Space, United Nations Publication A/AC.105/720, July 1999 